and practice development. Nurses and family carers are faced with different challenges when providing care to patients at the end of life. Therefore, it became imperative to explore the factors perceived to promote care at the end of life from the perspective of nurses, patients, and family carers.

Method A qualitative ethnographic case study design was employed for the study. Participant observations consisting of 188 hours of observation, and semi-structured interviews with 40 participants (nurses-14, patients-12, and family carers-14) were used as tools for data collection. Data analysis follows an inductive thematic approach using NVivo 11 qualitative data management programme.

Findings Six overarching themes emerged: Enhanced communication skills, Increase drug supply, improve manpower, reward or incentives, training of staff, and improve logistics supplies.

Conclusion Basically, these factors were directly related to the poor managerial processes and hospital's policies. If the hospital management increases drug supply, improve manpower, revive decayed infrastructure, improve logistics supplies, provide reward or incentives to staff, and encourages staff training and development, certainly the quality of palliative and end of life care will be improved.

\section{AN ANALYSIS OF THE QUALITY OF ADVANCED CARE PLAN AND DNACPR DOCUMENTATION PRIOR TO THE INTRODUCTION OF THE RESPECT PROCESS}

Kiran Parmar, Emma Wilkinson, Sinead Henderson, Clare Smith. St Peter's Hospital, Ashford and St Peter's Hospitals NHS Foundation Trust, Chertsey, Surrey

10.1136/bmjspcare-2019-ASP.81

An Advanced Care Plan (ACP) allows discussion and documentation of patient preferences for their future medical care which is not limited to resuscitation only. Despite national guidance, there remains poor communication and inconsistent documentation of ACP and DNACPR decisions, potentially resulting in inappropriate treatment and hospital admissions for patients. ReSPECT (Recommended Summary Plan for Emergency Care and Treatment) is a newly introduced emergency care plan which aims to help support advanced care discussions and record recommendations. We aimed to assess the quality of ACP and DNACPR documentation at a UK District General Hospital before introducing the ReSPECT form.

Retrospective data was collected on all patients from two elderly care wards in March 2018 through case note review. Section headings on the ReSPECT form were used as the basis for collection. Data was also collected on ACP/DNAR decision communication on discharge documentation.

87 patients (52 male and 35 female). 25\% (22) had ACP and $72 \%$ (63) had DNACPR agreed. Those with DNACPR, $79 \%$ (50) had discussion documented in clinical notes, and $57 \%$ (36) on discharge documentation. Those with ACP agreed, 68\% (15) of discussions were documented in clinical notes, 95\% (21) had ACP on discharge documentation completed by junior doctors.

ACP and DNACPR decisions are individual to each patient and our initial data shows they are not always clearly documented. Interestingly, ACP decisions are found more on discharge summaries than recorded in medical notes. Discharge summaries are completed by junior doctors and therefore need support by clear senior decision making in the notes. This is currently missing for $27 \%$ of ACP decisions and highlights that discussions required for the ReSPECT process are not currently routinely documented. The ReSPECT form alongside web-based training was introduced in October 2018. The next step is to re-audit and assess its impact on documentation and communication.

\section{PLANNING AHEAD: RETURNING HOME ON VENTILATORY SUPPORT FOR END-OF-LIFE CARE}

Jessica Walding, Meg Williams, Joanne Evans, Edward Curtis, Alison Stevens. Aneurin Bevan NHS Trust

\subsection{6/bmjspcare-2019-ASP.82}

Background The Intensive Care National Audit and Research Centre UK (ICNARC) revealed that approximately 20\% of patients admitted to the Intensive Care Unit (ICU) will die there. End of life care decisions are a fundamental aspect of care on ICU, particularly given that $70 \%$ of the deaths that occur are following the withholding or withdrawal of life-sustaining treatments. Palliative care is increasingly accepted as an essential component of comprehensive care for critically ill patients. Most deaths are managed independently on the ICU, as such, patients preferred place of death (PPD) is not often explored or fully established.

Aims

1. To highlight the importance of end-of-life care and early palliative care team input to everyday practice and training for anaesthetists and intensivists.

2. To actively consider whether transfer home may be a component of end-of-life care for some patients.

Methods Our patient population are those inpatients receiving additional respiratory support on the ICU, non-invasive or invasive ventilation, who's expressed their PPD as home. We recognise that collaborative and innovative working between teams within the acute and community sector is key to facilitating a safe and efficient transfer home for end-of-life care for patients requiring ventilatory support.

Results At our hospital, we have facilitated two patient transfers from ICU to home for withdrawal of ventilatory support and end-of-life care. Following these transfers, a comprehensive discharge checklist has been implemented, with key focus to ensuring a safe but time efficient transfer.

Conclusions To conclude, we are not asserting that transfer home at end-of-life is desirable or feasible for all critically unwell patients. However, we want to highlight the important of exploring patient wishes at this crucial stage of patient care.

\section{A REVIEW OF CODE STATUS DOCUMENTATION IN PATIENTS REFERRED TO THE PALLIATIVE CARE INPATIENT CONSULT SERVICE}

Sheena Geoghegan, Fiza Warsi, Sara Kleinschmidt, Reid Hartmann, Douglas Smucker. The Christ Hospital, Cincinnati, Ohio

\subsection{6/bmjspcare-2019-ASP.83}

Background Inpatient Palliative Care consult services see a wide range of acutely admitted patients, many of whom are critically ill. Due to the nature of an acute admission, code status clarification or discussion is often overlooked and can affect patient's clinical course and outcome. 